\title{
Medialität als Schlüsselkonzept eines sprachbewussten Literaturunterrichts am Beispiel einer Märchen-Stunde
}

\author{
Fabian Wolbring
}

Angenommen: 12. Januar 2021 / Online publiziert: 4. Februar 2021

(C) Der/die Autor(en) 2021

Zusammenfassung Analog zur disziplinären Einteilung in Sprach-, Literatur- und Mediendidaktik werden Sprache, Literatur und Medien im Deutschunterricht häufig suggestiv zu unterrichtspraktisch separaten Phänomenen stilisiert. Der Beitrag wirbt dagegen für ein integratives Miteinander, bei dem die Sprache selbst offensiv als Medium be- und verhandelt wird, wobei insbesondere die ästhetisierte, entpragmatisierte Sprache der Literatur als Display sprachlicher Medialität fungieren kann (Zymner 2009). Nach einer einführenden theoretischen Skizzierung wird das Konzept am Beispiel einer Märchenstunde zum Rumpelstilzchen exemplifiziert. Auf der Suche nach heimlichen MitsprecherInnen erfahren die SuS hier, wie das Medium Sprache gleichzeitig eine Einstimmigkeit suggerieren und Mehrstimmigkeiten ermöglichen kann.

Schlüsselwörter Medienreflexionskompetenz - Sprache als Medium · Medialität · Rumpelstilzchen · Mehrstimmigkeit · Heimliche MitsprecherInnen

F. Wolbring $(\bowtie)$

Neuere deutsche Literatur und Didaktik, Universität Marburg, Marburg, Deutschland

E-Mail: fabian.wolbring@uni-marburg.de 


\title{
Mediality as a Key Concept in the Language-Aware Teaching of Literature Exemplified in a Fairy Tale-Lesson
}

\begin{abstract}
In analogy to the disciplinary division into language, literature and media didactics, language, literature and media are often suggestively stylized as separate phenomena in the practical teaching of German. This paper, on the other hand, advocates an integrative approach in which language itself is proactively treated and negotiated as a medium, whereby in particular the aestheticized, depragmatized language of literature can function as a display of linguistic mediality (Zymner 2009). After an introductory theoretical outline, the concept will be exemplified using a German literature lesson on the fairy tale Rumpelstiltskin. In their search for hidden co-speakers, the students learn how the medium of language can simultaneously suggest unanimity and enable polyphony.
\end{abstract}

Keywords Media Reflection Competence - Language as a Medium · Mediality · Rumpelstiltskin $\cdot$ Polyphony $\cdot$ Hidden Co-Speakers

\section{Einführende theoretische Skizzierung}

Die akademische Spaltung der Germanistik in Linguistik und Literaturwissenschaft lässt sich auch im Deutschunterricht beobachten. Schulcurricula tendieren zu einer möglichst paritätischen Aufteilung in sprach- und literatur-orientierte Reihen, wobei den sprachunterrichtlichen Segmenten nicht selten die Aufgabe zukommt, normgerechten Sprachgebrauch zu vermitteln, ${ }^{1}$ während der Literaturunterricht tendenziell inhaltistisch geprägt ist, also zur Verhandlung (vermeintlich) relevanter Themen wie Freundschaft, Frühling oder Fremdheit dient, ohne dass die spezifische Darstellungsweise notwendig größere Beachtung fände. Sprachreflexion wird dabei häufig als genuines Lernziel des Sprach- bzw. Grammatikunterrichts angesehen, während dem Literaturunterricht zuweilen eine regelrechte Sprachvergessenheit attestiert werden kann. Der Literaturdidaktiker Hans Lösener mahnt entsprechend:

Eine zentrale Herausforderung für die didaktische Weiterentwicklung des literarischen Lernens liegt deshalb in der Erforschung und Beschreibung der spezifischen Sprachlichkeit des literarischen Lernens. Denn literarisches Lernen ist sprachliches Lernen. ${ }^{2}$

\footnotetext{
1 Vgl. hierzu etwa die Einschätzung von Abraham und Kepser: »Schulische Sprachbetrachtung erfolgt oft immer noch als Grammatikunterricht, in dem Beschreibungskategorien der tradierten Lateingrammatik vermischt mit solchen einer funktionalen Grammatik gelehrt werden« (Abraham und Kepser 2016 [2005], S. 123). Vgl. ebenso aus dem Kerncurriculum der gymnasialen Oberstufe des Landes NRW: »Reflexion über Sprache bezieht sich auf Fragen des formalrichtigen und funktional adäquaten Umgangs mit Sprache, auf die Komplexität und Vielfalt der sprachlichen Ausdrucksformen sowie auf die historischen Wandlungsprozesse der deutschen Sprache« (Ministerium für Schule und Weiterbildung des Landes Nordrhein-Westfalen 2014, S. 17).
}

2 Lösener 2015, S. 73. 
Einer solchen Einteilung in Sprach- und Literaturunterricht gemäß erscheinen auch Sprach- und Literaturdidaktik als separate Disziplinen. Hinzu tritt vermehrt eine dritte, scheinbar distinkte Akteurin, nämlich die Mediendidaktik. Sprache, Literatur und Medien werden so häufig suggestiv zu unterrichtspraktisch separaten Phänomenen stilisiert.

M.E. wäre es indes sinnvoll und sachgerecht, gerade den Medienbegriff im Deutschunterricht so weit auszuweiten und so zu disponieren, dass die unterrichtliche Auseinandersetzung mit Texten - insbesondere mit literarischen - als ebenso fundamentaler wie genuiner Beitrag zur vielbeschworenen Medienkompetenz erkannt bzw. genutzt wird. Medialität stellt ein Konzept dar, das unmittelbar an vertraute Vorstellungen der SuS anknüpft und gleichzeitig geeignet scheint, eben diese Denkroutinen zu irritieren und Vorstellungsmuster zu differenzieren. In der Medientheorie und -philosophie avancierte der Medialitätsbegriff durch Arbeiten von Ludwig Jäger, ${ }^{3}$ Sybille Krämer, ${ }^{4}$ Martin Seel ${ }^{5}$ oder Jan Georg Schneider ${ }^{6}$ im Laufe des letzten Jahrzehnts zu einem heuristischen Schlüsselbegriff. Wie genau Informationen sprachlich mediatisiert und kognitiv reaktualisiert werden, bleibt dabei natürlich eine längst noch nicht final beantwortbare Forschungsfrage, die in der Lesedidaktik ${ }^{7}$ genauso diskutiert wird wie in der Rezeptionsforschung, ${ }^{8}$ der Erkenntnistheorie, ${ }^{9}$ den Kognitionswissenschaften, ${ }^{10}$ der Erzähltheorie, ${ }^{11}$ der Semiotik, ${ }^{12}$ der Semantik, ${ }^{13}$ der Pragmatik, ${ }^{14}$ der Rhetorik, ${ }^{15}$ der Poetik, ${ }^{16}$ der klassischen Informationstheorie ${ }^{17}$ und diverser anderer kultur- und sprachwissenschaftlicher Disziplinen. Zur Erarbeitung schulpraktisch operationalisierbarer Begriffe und Konzepte zur Sensibilisierung für sprachliche Medialität kann auf ein entsprechend breites Korpus an Forschungsliteratur rekurriert werden, ohne dass eine Letztbeweisbarkeit erwartet oder auch nur angestrebt werden müsste. Eine schulpraktische Adaption kann nicht den Anspruch formulieren, endgültige Antworten auf strittige epistemologische Fragen zu generieren. Viel eher geht es darum, SuS als intuitive und durchaus auch virtuose Sprachverwender mit Fragen der eigenen Sprachrezeption wie des eigenen Sprachgebrauchs zu konfrontieren und sie so zu befähigen, auf dem Niveau ambitionierter Laien introspektiv metasprachliche und damit metakognitive Beobachtungen anzu-

\footnotetext{
3 Vgl. etwa Jäger 2015.

4 Vgl. etwa Krämer 2004.

5 Vgl. etwa Seel 1998.

6 Vgl. etwa Schneider 2008.

7 Vgl. etwa Rosebrock und Nix 2008.

8 Vgl. etwa Iser 1984 [1976].

9 Vgl. etwa Maturana 1982.

10 Vgl. etwa Fauconnier und Turner 2002.

11 Vgl. etwa Willand 2014.

12 Vgl. etwa Eco 1972 [1968].

13 Vgl. etwa Zimmermann 2014.

14 Vgl. etwa Searle 1990 [1979].

15 Vgl. etwa Dockhorn 1968.

16 Vgl. etwa Stockwell 2002.

17 Vgl. etwa Shannon und Weaver 1948.
} 
stellen und diese mit denen ihrer MitschülerInnen zu vergleichen. Hierzu gilt es ein funktionales Analyseinstrumentarium zu entwickeln und zu didaktisieren.

Sprache als Medium zu betrachten, erscheint gerade im Schulkontext nicht selbstverständlich. Dabei lässt sie sich sogar plausibel als alleiniges Leit-, Ur- oder Archimedium beschreiben. ${ }^{18}$ Der Kultur- und Medienwissenschaftler Ludwig Jäger moniert entsprechend auch die Sprachvergessenheit der Medientheorien und weist wiederholt auf den archetypischen Charakter der Sprache für jedweden Prozess der Mediatisierung hin. So sei »bislang kein Medialitätsstatus fortgeschrittener Mediengesellschaften denkbar, der nicht systematisch auf die anthropologische Medialität der Sprache zurückbezogen bliebe. $\ll^{19}$

Im rezenten Bildungsdiskurs wird Medienkompetenz allzu häufig auf Bedienfähigkeiten enggeführt; insbesondere bezogen auf digitale Apps und Gadgets. ${ }^{20}$ Dabei kann gerade in der unterrichtlichen Verhandlung und Reflexion des Digitalen eine medienbewusste Bezugnahme auf das Proto-Medium Sprache sehr ertragreich sein. Das Digitale erscheint so nicht mehr zwangsläufig als das radikal neue und andere, sondern als etwas, das in seiner technisch-medialen Anlage in der Sprache präfiguriert ist und in seiner medialen Ausgestaltung in ähnlicher Weise sinnfällig wird wie andere Medien zuvor. Medienkompetenz wird so offensiv als Medienreflexionskompetenz spezifiziert, mit dem Bildungsauftrag, Vermittlungsprozesse sichtbar zu machen, Denkroutinen zu deautomatisieren und die medienbedingte Genese von Vorstellungen nachzuvollziehen.

Die Medialität der Sprache, d.h. ihre genuinen operativen Verfahren zur Vermittlung und Generierung von Information, ist dabei bekanntermaßen besonders eng mit unserem Denken verzahnt. In der Sensibilisierung für diese Medialität liegen entsprechend große Bildungspotentiale für den Deutschunterricht. Sie schult eine Sprachbewusstheit weit über den normgerechten Sprachgebrauch hinaus.

Dabei stellt der Gebrauch von Sprache bekanntermaßen eine im Grunde höchst anspruchsvolle Medientechnik dar, die den SuS (wie wohl beinahe allen Menschen) aber so selbstverständlich vertraut ist, dass sie sie nicht als solche wahrnehmen. Die Sichtbarmachung der sprachlichen Medialität führt folglich zu einer - kognitiv höchst anregenden - Irritation. Der in der Schule üblicherweise forcierte Erkenntnisweg des Vertrautmachens von Fremdem wird invertiert zu einem - sicher nicht weniger ergiebigen - Fremdmachen von Vertrautem, worin ein potentiell profilschärfendes Alleinstellungsmerkmal des Deutschunterrichts im Fächerverbund liegt.

Womöglich eignet sich die Bezugnahme auf die Medialität der Sprache zukünftig als sinnstiftende universale Klammer bzw. als Dispositiv für alle im Deutschunterricht verhandelten Inhaltsfelder und Kompetenzbereiche. Dem Literaturunterricht kommt dabei m.E. eine essentielle Rolle zu, denn Literatur ist eine Form der Sprachverwendung, die die Spezifika dieses Mediums in besonderer Weise ausstellt und

\footnotetext{
18 Vgl. Ehlich 1998, S.20.

19 Jäger 2001, S. 22.

20 In der Strategie der Kultusministerkonferenz zur Bildung in der digitalen Welt füllt die Analyse und Reflexion von Medien entsprechend nur einen von sechs Kompetenzbereichen aus (Kultusministerkonferenz 2017 [2016]).
} 
veranschaulicht. Gerade die ästhetisierte, entpragmatisierte Sprache der Literatur kann als Display sprachlicher Medialität fungieren, ${ }^{21}$ deren Erschließung ein enges Zusammenspiel von Sprach- und Literaturdidaktik voraussetzt. Über den Medienbegriff kann der Literaturunterricht dem Verdacht der Lebensferne begegnen, ohne seine Gegenstände und seine formanalytische Ausrichtung notwendig aufgeben $\mathrm{zu}$ müssen. Im Gegenteil erscheinen gerade die vielfältigen Analysemethoden des Literaturunterrichts zur Beschreibung von Form und Ästhetik prädestiniert dazu, als Werkzeuge zur Erfassung von Vermittlungsprozessen eingesetzt zu werden. Sie liefern bei minimaler Neujustierung bereits ein unterrichtspraktisch bewährtes Instrumentarium für Reflexion über sprachliche Medialität am konkreten Text.

\section{Kennzeichen der Medialität der Sprache}

Technisch ist die Medialität der Sprache bestimmt durch die Semiose symbolischer Zeichen, d.h. Information wird vermittelt bzw. generiert durch die Aktualisierung bekannter, artifizieller, konventionalisierter und reglementierter Zeichen. ${ }^{22} \mathrm{Zu}$ ihren basalen Anordnungsprinzipien gehört etwa die Tendenz zur Einstimmigkeit wie auch die Linearität, die bedingen, dass Information stets sukzessive, in Form aneinandergereihter «Bedeutungs-Chunks« vermittelt wird. Der Sprache entnommene Information zeichnet dabei eine bemerkenswerte Immaterialität und situative Entbundenheit aus, die weltlicher Erfahrung gänzlich abgeht und die dazu führen kann, dass RezipientInnen versuchen, die Information selbstdenkend situativ zu verorten. In der kognitiven Verarbeitung regt die Sprache Denkgewohnheiten an, die teils auf individuelle Erfahrungs- und Verarbeitungsroutinen zurückzuführen sind, teils auf kulturell etablierte Frames und Scripts, Topoi und Narrative, wie auch auf »Gattungswissen« über bestimmte Typen von Äußerungen. Sprachlich vermittelte Information tendiert daher auch zu Standardisierung und damit Vereinheitlichung. Sie gilt, ihrer Immaterialität zum Trotz, aufgrund ihrer begrifflichen Präzision als sehr verbindliches Medium, dem häufig ein Wirklichkeitsbezug unterstellt wird. Auch wirkt sie in ihrer alteritären Ausrichtung inhärent appellativ.

Den Sprachzeichen werden (häufig unbewusst) diverse Informationsdimensionen unterstellt, d.h. es werden Rückschlüsse gezogen, die in der Regel faktisch nicht endgültig zu verifizieren sind und die doch intuitiv wahrscheinlich erscheinen und dabei durchaus problematisch sein können; z.B. ${ }^{23}$

Es handelt sich um eine Äußerung, d.h.

- sie wurde von jemandem geäußert,

- sie wurde bewusst und intentional geäußert,

- sie wurde von einer einzelnen Person geäußert,

\footnotetext{
21 Vgl. Zymner 2009, S. 139.

22 Vgl. etwa Peirce 1983 [1903], de Saussure 2001 [1916] oder Eco 1972 [1968].

23 Die hier aufgeführte Liste stellt eine lose Heuristik ohne Anspruch auf Vollständigkeit dar und orientiert sich in der Anlage an den Konversationsmaximen von Grice (1975).
} 
- sie wurde von einer deutschsprachigen Person geäußert (womöglich einer weißen, männlichen, erwachsenen etc.),

- sie wurde in einer bestimmten Situation geäußert,

- die in der Äußerung enthaltenen Informationen sind wahr bzw. die äußernde Person geht davon aus, dass sie wahr sind,

- die in der Äußerung enthaltenen Informationen sind für potentielle RezipientInnen relevant bzw. die äußernde Person geht davon aus, dass sie relevant sind (Äußerungswürdigkeit),

- die in der Äußerung enthaltenen Informationen sind ausreichend vollständig bzw. die äußernde Person geht davon aus, dass sie ausreichend vollständig sind,

- das in der Äußerung verhandelte Geschehen entspricht einem lebenswirklichen Geschehen,

- etc.

Unterstellungen dieser Art gilt es zu reflektieren. Der Vermittlungsprozess soll »gestört « und damit sichtbar gemacht werden. Die SuS sollen eine gesunde Skepsis gegenüber ihrem primären Verständigungsmedium entwickeln.

\section{Didaktisierung}

Reflexion über sprachliche Medialität ist grundsätzlich in allen Jahrgangsstufen sinnvoll, da SuS in der Regel bereits selbstverständliche und intuitive SprachnutzerInnen sind, die einträglich nach ihren Spracherfahrungen befragt werden können. Zum basalen methodischen Repertoire gehört dabei die Erhebung klasseninterner Daten zum Textverständnis. Sie dient dazu, jeweils probabilisitische Lesewege zu erschließen, die anschließend plausibilisiert werden können. Darüber hinaus gilt es, vielfältige Übungen zur Paraphrase und beobachtenden Umformulierung von Texten einzusetzen, die für die Wirkung spezifischer Formulierungen sensibilisieren. Auch Vergleiche der eigenen Textwahrnehmung mit medialen Umsetzungen von Film, Bild, Theater, Comic, Computerspiel, usw. sind sinnvoll, da sie helfen der spezifischen Medialität von Sprache analytisch habhaft zu werden.

Zur Veranschaulichung soll das folgende unterrichtspraktische Beispiel für die Klassenstufen 8/9 dienen. Die SuS untersuchen dabei eine Passage aus dem Märchen Rumpelstilzchen, erkennen, dass ein Satz gleichzeitig mehrere UrheberInnen und damit MitsprecherInnen haben kann und reflektieren deren jeweils unterschiedliche Motive. Dadurch erlernen sie einen quellenkritischen Umgang mit dem Medium Sprache und erweitern so ihre Medien- und Sprachreflexionskompetenz.

\section{Medienreflexionskompetenz an Märchen ausbilden}

Die Forderung nach mehr Medienkompetenz ist im Bildungsdiskurs nahezu allgegenwärtig. Dass nun allerdings gerade in der Verhandlung literarischer Klassiker wesentliche Potentiale liegen, die Medienreflexion von Kindern und Jugendlichen des digitalen Zeitalters maßgeblich zu verbessern, vermuten wohl die wenigsten. 
Den Märchen der Brüder Grimm - zumindest den populärsten unter ihnen - kommt dabei womöglich eine Sonderrolle zu, da sie zugleich als historische Klassiker gelten und doch weiterhin intermedial adaptiert, reaktualisiert und verbreitet werden und entsprechend die Kinder-, Jugend- und Populärkultur bis in die unmittelbare Gegenwart mitbestimmen. Diese mediale Mobilität scheint der Gattung geradezu eingeschrieben zu sein: Märchen wurden und werden erzählt und wiedererzählt und dabei auch variiert, modernisiert und nicht selten instrumentalisiert. Da sie aber als zeitlose Klassiker erscheinen und viele ihrer erzählerischen Charakteristika behalten, werden die Veränderungen und der Bedeutungswandel, die mit jeder Reaktualisierung unweigerlich einhergehen, häufig übersehen, ebenso wie der Umstand, dass mit jedem Neu- und Wiedererzählen neue Sprechinstanzen hinzutreten, die eigene Motive und Interessen verfolgen.

Rumpelstilzchen ist nun allerdings ein Märchen, das nicht nur beständig neuund wiedererzählt wird, sondern das das Wiedererzählen als handlungsrelevanten Akt selbst vorführt. Würde der Bote die berühmten Erkennungsverse des Rumpelstilzchens nämlich nicht weitererzählen, könnte die Müllerstochter offensichtlich auch den erlösenden Namen nicht raten. Im grimmschen Originaltext wird dabei bemerkenswerterweise sogar nur das Wiedererzählen des Boten geschildert. Allerdings fällt dieses durch das wörtliche Zitat so plastisch aus, dass es in der Rezeption kaum bewusst wird. Aus diesem Grund eignet sich die Stelle ideal zur Reflexion von heimlicher Mehrstimmigkeit.

\section{Heimliche MitsprecherInnen erkennen}

Wer einen Satz liest oder hört, geht in der Regel davon aus, dass dieser von einer und nur einer Äußerungsinstanz stammt. Das Medium Sprache ist sowohl in der Mündlichkeit wie auch in der Schriftlichkeit durch eine starke Tendenz zur Einstimmigkeit geprägt. Der menschliche Sprechapparat ist nicht dazu angelegt, gleichzeitig mehrere Lautzeichen erklingen zu lassen; unser Schriftsystem reiht seine Zeichen analog dazu stets sukzessive nacheinander. Dabei übersehen wir allerdings leicht, dass uns eine Äußerung häufig nicht unmittelbar durch ihre Urheberinstanz erreicht, sondern als weitererzählte und medial aufbereitete Reproduktion. Neben die ursprüngliche Sprechinstanz treten so heimliche MitsprecherInnen, denen man produktiv nachspüren kann. Die markanten Verse in unserem Beispiel erkennen wir eindeutig als die Äußerung Rumpelstilzchens, doch sind sie gleichzeitig auch die Äußerungen des Boten, der Erzählinstanz, der Brüder Grimm und all derjenigen, die sie in der Vergangenheit rezitiert haben und in Zukunft rezitieren werden, denn in jeder Reaktualisierung verbinden und vermengen sich (unmerklich) die unterschiedlichen Äußerungssituationen, -perspektiven und -motive. Es kann unterrichtspraktisch hoch produktiv sein, die SuS für diese Mehrstimmigkeit zu sensibilisieren, mit ihnen nach Spuren von heimlichen MitsprecherInnen zu suchen und dabei kulturgeschichtliche Tiefenbohrungen zu unternehmen. 


\section{Intention}

Gerade im digitalen Zeitalter gilt es, SuS dazu zu befähigen, Medien nicht nur zu konsumieren, sondern zu hinterfragen und zu reflektieren. Die medialen Einwirkungen auf Kinder und Jugendliche sind enorm hoch. Beinahe scheint es, als würden kaum noch unmittelbar lebensweltliche Erfahrungen gemacht, die nicht medienvermittelt auf sie eindringen. Die Auseinandersetzung mit der heimlichen Mehrstimmigkeit von medial vermittelten Äußerungen ermöglicht es den SuS, sich für die verschiedenen Intentionen von informationsverbreitenden Instanzen zu sensibilisieren und erweitert so ihre Medienreflexionskompetenz. Das skizzierte Unterrichtsmodell für die Jahrgangstufe 8/9 will bewusst einen scheinbar vertrauten und für die Altersgruppe reizlosen Gegenstand neu verhandeln und die Lerngruppe zunächst suggestiv in die Irre führen. Ihre Überzeugung, den Urheber der bekannten Verse zu kennen, wird irritiert und so ein kognitiver Konflikt initiiert. Dadurch verdeutlicht das Modell, wie aufmerksame, kritische und historisch fundierte Lesewege dem Bekannten neue Denkimpulse abgewinnen können. Die SuS üben dadurch, bei der Rezeption von Medien aufmerksam zu sein und voreilige Schlüsse zu vermeiden.

Die analytische Herangehensweise hilft den SuS in ihrer Entwicklung zu mündigen MedienrezipientInnen, schärft ihr Sprachbewusstsein und fungiert als Propädeutikum für die Erzähltextanalysen in der Oberstufe.

Die Arbeit mit Faksimiles der Originaltexte hilft den SuS, Texte als physische Medienprodukte wahrzunehmen und intuitiv historisch zu verorten. Gleichzeitig wird durch die ungebrochene Aktualität des Gegenstandes ein unmittelbarer Lebensweltbezug hergestellt. Dieser kann und sollte im weiteren Verlauf der Reihe durch Bezüge auf aktuelle mediale Adaptionen des Märchens nach Möglichkeit noch vertieft und fokussiert werden [s. Ausblick].

Das Modell ist prinzipiell in unterschiedlich gelagerten Unterrichtsreihen für die Mittelstufe realisierbar. Offensichtlich kann es in Märchen- und andere erzähltextanalytische Reihen integriert werden; zudem kann es aber auch dezidiert als Bestandteil von sprach- und medienreflexiv ausgerichteten Reihen fungieren.

\section{Einstieg}

Zum Einstieg konfrontiert man die Lerngruppe mit einer Projektion ${ }^{24}$ der berühmten Verse aus dem Märchen Rumpelstilzchen. Dabei wird bewusst mit einem Faksimile gearbeitet; zum einen, um die Zeilen auf ihren Ursprung in der grimmschen Originalausgabe und damit als Botenzitat festzulegen (in anderen Versionen des Märchens werden sie zuweilen nicht als reines Zitat erzählt), zum anderen, um eine erste mediale Spur zum historischen Kontext der Erstveröffentlichung zu legen. Unter dem Vorwand einer Vorwissensabfrage erhalten die SuS die Aufgabe, die projizierte Abb. 1 auszufüllen, also Urheber, Äußerungskontext und potentielle Äußerungsmotive anzugeben und die Ergebnisse anschließend im offenen Unterrichtsgespräch auszutauschen. In Anbetracht der hohen Verbreitung des Märchens

${ }^{24}$ Das Modell kann sowohl über Folien als auch über digitale Projektion realisiert werden. 


\begin{tabular}{|c|c|}
\hline & 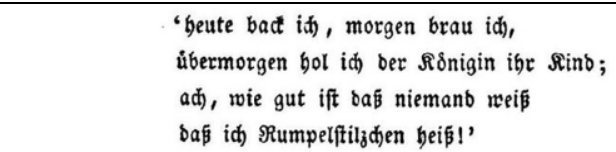 \\
\hline $\begin{array}{l}\text { Wer spricht } \\
\text { diese Sätze? }\end{array}$ & \\
\hline $\begin{array}{l}\text { In welchem } \\
\text { Kontext } \\
\text { werden sie } \\
\text { geäußert } \\
\text { (wann / } \\
\text { wo)? }\end{array}$ & \\
\hline $\begin{array}{l}\text { Warum } \\
\text { werden sie } \\
\text { gesagt? }\end{array}$ & . \\
\hline
\end{tabular}

Abb. 1 Tabelle

und der Tatsache, dass Märchen bereits in früheren Jahrgangsstufen verhandelt wurden und die SuS dem typischen Märchenalter längst entwachsen sind, ist davon auszugehen, dass der Großteil der Lerngruppe die Aufgabe als reizlos und unterfordernd empfindet. Die Frage nach den Gründen für die Äußerung kann allerdings durchaus kontrovers diskutiert werden, da nicht unbedingt klar ist, ob die Äußerung als Triumphgesang, Beschwörungsformel oder (manisches) Selbstgespräch zu deuten ist und die Figur folglich ihre im gesamten Text etablierte Rätselhaftigkeit behält. SpötterInnen mögen an diesem Punkt womöglich schon darauf hinweisen, dass die Äußerung vor allem dadurch motiviert sein könnte, die Geschichte zur Auflösung zu bringen. Nachdem die Ergebnisse abgeglichen wurden, werden sie von der Lehrkraft konsequent verneint, wodurch eine produktive Irritation erreicht wird, die die SuS für die folgende Erarbeitungsphase motiviert.

\section{Erarbeitung 1}

Nachdem die Lerngruppe in dieser Weise irritiert wurde, soll der Faksimileausschnitt durch einen größeren ersetzt werden, der die gesamte Buchseite abbildet (Abb. 2). Dieser sollte zur besseren Lesbarkeit in Form eines Handouts verteilt werden und Grundlage einer genaueren Untersuchung durch die SuS bilden. Trotz der Widerstände, die die Fraktur beim Lesen bereiten dürfte, wird die Pointe der Aufgabe voraussichtlich schnell klar: Nicht Rumpelstilzchen selbst spricht die Verse, sondern der Bote zitiert es. Die projizierte Tabelle wird nun um eine weitere Spalte ergänzt (Abb. 3), in die der Bote als Urheber eingetragen wird. Die SuS übertragen die bisherigen Ergebnisse und füllen nun im gegenseitigen Austausch die neu hinzugefügte Spalte (Bote) aus (Abb. 4). Äußerungskontext und Motive sind nun völlig andere, da es sich plötzlich um einen ganz anderen Sprechakt handelt: Statt eines euphorischen Triumphgesangs lesen wir nun einen Botenbericht mit informierender Absicht. Entscheidend für die Medienreflexionskompetenz ist nun, dass den $\mathrm{SuS}$ in der Diskussion und Sicherung dieser Ergebnisse deutlich wird, dass dieselbe 
Abb. 2 Faksimile der originalen Buchseite (Quelle: Brüder Grimm: Kinder- und Haus-Märchen. Große Ausgabe. Band 1, 2. Auflage, Berlin 1819, S. 336)

$$
-336-
$$

lein 'To beiß iđ nid).' Den zweiten Tag ließ fie herumfragen

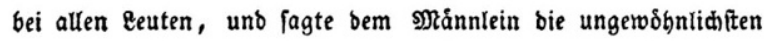
und feltjamften vor, Rippenbieft, Sammetrwabe, Sdnirbein, aber eछ blieb babei 'fo heis id nidtt.' Den britten Iag fam

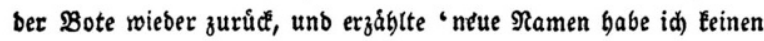
einzigen finden tónnen, aber wie id) an einen bohen Berg um

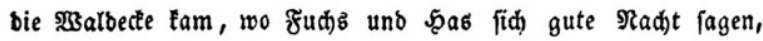
fo fah id) ba ein fleines Scaus, und vor bem Şaus brannte ein Feuer, uno um bas Feuer fprang ein gar zu låderlides Mánnden, bưpfte auf einem Rein, und farie

'Geute bact id), morgen brau id),

úbermorgen Gol idh ber Rosnigin igr Rinb;

ad), wie gut ift baß niemanb weiß

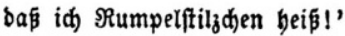

Da war bie. Ronigin ganz frog baß fie ben Ramen ruste, unb als balb bernad) bas Mannlein fam, unb iprad) 'nun, frau

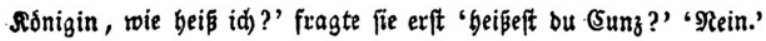
' Đeikeft bu ફ̧einz?' 'গein.'

'ફeißjt bu etwa Rumpelftilzd)en?'

'Das hat bir ber Teufel gefagt, dab hat bir ber Reufel gefagt' (d)rie bas Månntein, und ftiés mit bem redsten fü bor 3orn fo'tief in bie Erbe baß es bis an ben \&eib hineinfugr, bann

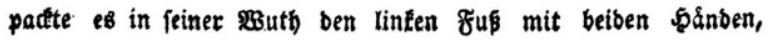
und riß fid felbft mitten entionei.

Textstelle gleichzeitig beide Sprechakte manifestiert und beide für den Handlungsverlauf des Märchens entscheidend sind. Ist dies gewährleistet, kann die Lerngruppe mit dem Hinweis auf einen weiteren Mitsprecher abermals irritiert werden.

\section{Erarbeitung 2}

Sollte in der Lerngruppe niemand darauf kommen, welcher Mitsprecher gemeint ist, kann die Lehrkraft mit der Frage »Wer spricht denn den Satz: Den dritten Tag kam der Bote wieder zurück, und erzählte?« nachhelfen. In Anknüpfung an ihr Vorwissen sollten die SuS die gegebene Erzählinstanz als »auktorialen« oder »allwissenden Erzähler « klassifizieren und erkennen, dass eigentlich dieser erzählt, wie der Bote erzählt, was das Rumpelstilzchen gesprochen hat; folglich also drei distinkte Stimmen gleichzeitig erklingen. Zur besseren Vergegenwärtigung dieser Mehrstimmigkeit kann dieser Befund in einem kurzen Rollenspiel nachvollzogen werden: Die SuS machen Vorschläge, wie der ursprüngliche Textausschnitt im Ton der jeweiligen Sprechinstanz zu lesen ist, wobei durchaus unterschiedliche Vorstel- 


\begin{tabular}{|c|c|c|}
\hline \multicolumn{3}{|c|}{ 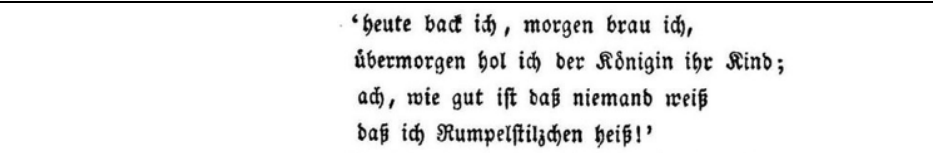 } \\
\hline $\begin{array}{l}\text { Wer spricht } \\
\text { diese Sätze? }\end{array}$ & Rampebstizrohen & Bote \\
\hline $\begin{array}{l}\text { In welchem } \\
\text { Kontext } \\
\text { werden sie } \\
\text { geäußert } \\
\text { (wann / } \\
\text { wo)? }\end{array}$ & & \\
\hline $\begin{array}{l}\text { Warum } \\
\text { werden sie } \\
\text { gesagt? }\end{array}$ & & \\
\hline
\end{tabular}

Abb. 3 Tabelle (erweitert)

\section{Arbeitsaufträge}

1. Übertrage die Ergebnisse für die Spalte Rumpelstilzchen.

2. Fülle die zweite Spalte für den Boten nun selbst aus. EA

3. Tausche dich mit deiner Nachbarin / deinem Nachbarn über eure Ergebnisse aus. Ergänzt ggf. eure Spalten. PA

\begin{tabular}{|c|c|c|}
\hline & $\begin{array}{l}\text { 'Geute bact } \\
\text { úbermorgen } \\
\text { ad), wie gu } \\
\text { baß̧ id) Run }\end{array}$ & \\
\hline $\begin{array}{l}\text { Wer spricht } \\
\text { diese Sätze? }\end{array}$ & Rampebstizchen & Der Bote \\
\hline $\begin{array}{l}\text { In welchem } \\
\text { Kontext } \\
\text { werden sie } \\
\text { geäußert } \\
\text { (wann / } \\
\text { wo)? }\end{array}$ & & \\
\hline $\begin{array}{l}\text { Warum } \\
\text { werden sie } \\
\text { gesagt? }\end{array}$ & & \\
\hline
\end{tabular}

Abb. 4 Handout zur ersten Erarbeitungsphase 


\section{Arbeitsaufträge}

1. Überlege, wieso die Erzählinstanz die Verse des Rumpelstilzchen vom Boten sprechen lässt. Halte deine Überlegungen in der obersten Spalte fest.

2. Gib dann dein Blatt an deine linke Nachbarin / deinen linken Nachbarn weiter und nimm den Zettel deiner rechten Nachbarin/deines rechten Nachbarn entgegen. Kommentiere das, was er/sie geschrieben hat.

3. Wenn du deinen eigenen Zettel zurückbekommst: Lies dir die Kommentare deiner MitschülerInnen durch. Verfasse ein Fazit.

Die Erzählinstanz hat die Verse vom Boten sprechen lassen, weil...

Kommentar 1:

Kommentar 2:

Kommentar 3:

Mein Fazit:

Abb. 5 Handout zur zweiten Erarbeitungsphase in Gruppendiskussion 


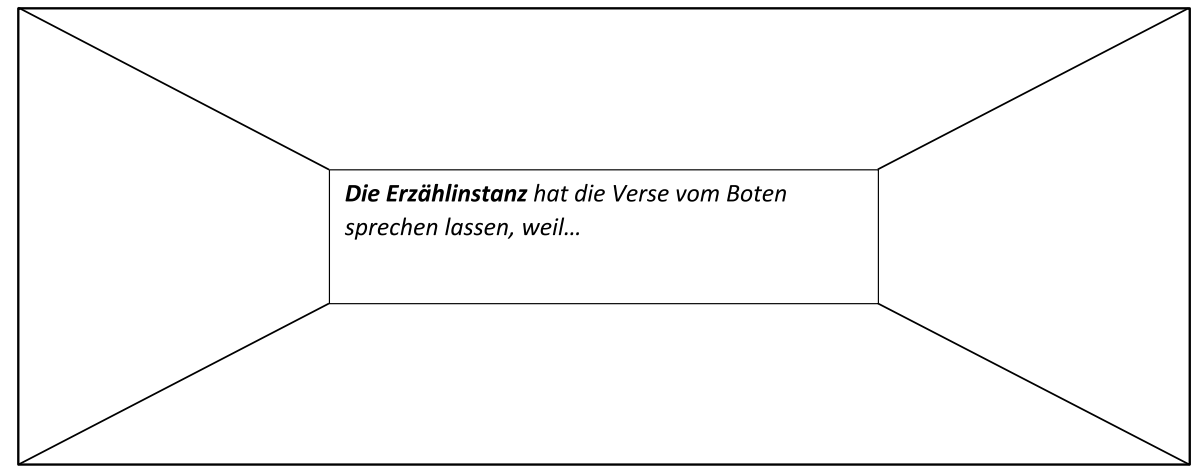

Abb. 6 Vereinfachte Placemat zur zweiten Erarbeitungsphase

lungen plausibel erscheinen (Rumpelstilzchen könnte die Verse etwa genauso gut euphorisch wie beschwörend sprechen, der Bote ebenso nüchtern wie aufgeregt berichten, die Märchenerzählinstanz emotional distanziert oder involviert erzählen). Nun sollte der Text von drei unterschiedlichen SuS in der jeweiligen Rolle ein paar Mal gleichzeitig gesprochen werden, um den Effekt der Mehrstimmigkeit zu verdeutlichen.

Dabei gilt es, der Lerngruppe diesen Befund als durchaus bemerkenswerte Darstellungsstrategie des Märchens zu verdeutlichen. Schließlich wird die bekannteste Szene des Märchens, d.h. der Tanz des Rumpelstilzchens um das Feuer und die belauschte Versrede, nicht »direkt« von der Erzählinstanz erzählt und ist somit eigentlich gar nicht Teil des »unmittelbar« geschilderten Erzählgeschehens.

Die SuS sollen sich diese Darstellungsweise nun erklären, indem sie ergebnisoffen der Frage nachgehen, warum der Text die Ereignisse im Wald und im Schloss nicht separat, sondern über den Botenbericht gleichzeitig erzählt. Hierzu bietet sich etwa eine Gruppendiskussion (Abb. 5) oder vereinfachte Placemat-Methode (Abb. 6) an. Mögliche Hypothesen zur Darstellungsstrategie des Textes könnten lauten: Der Text ist dadurch kürzer und muss die Ereignisse im Wald nicht doppelt erzählen. Der Text ist dadurch plastischer und unmittelbarer als wenn die Ereignisse im auktorialen Erzählton geschildert worden wären. Der Text erhält durch den Botenbericht eine mögliche Unzuverlässigkeit und behält eine Spannung für die Auflösung, usw.

Zur Präsentation und Sicherung der Ergebnisse böte sich der Einsatz einer Dokumentenkamera an, wie auch ein an die Erarbeitungsphase anschließender Museumsgang. Ein möglicher Grund, der sich den SuS wohl nur schwerlich erschließt und in der Sicherung der Ergebnisse ergänzt werden kann, liegt in der übergeordneten Perspektivierung des Märchens: So bleibt das Geschehen stets an den Erfahrungshorizont der Müllerstochter gekoppelt und folgt weder dem Rumpelstilzchen noch dem Boten unmittelbar in andere Settings. Dies begünstigt auch die subtile Sympathielenkungsstrategie des Textes, nach der das Rumpelstilzchen stets als fremd markiert wird, da seine Motive und Handlungen nicht durch die Erzählinstanz erklärt werden. Unabhängig davon, wie sich die SuS die Darstellungsweise genau plausibilisieren, sensibilisieren sie sich für die Präsenz heimlicher Mitsprecher in der medialen Verbreitung von Informationen. Diese Medienreflexionskompetenz kann und sollte in der Folge noch ausgeweitet werden. 


\section{Ausblick}

Die Spurensuche nach heimlichen MitsprecherInnen kann sowohl in die Vergangenheit, als auch in die Gegenwart weiterverfolgt werden. Im Falle der grimmschen Märchen bietet es sich etwa an, die Rolle der Grimms als Herausgeber und Philologen zu untersuchen, um die SuS auf den Akt des Herausgebens als eigenen (heimlichen) »Äußerungsakt« im Produktionsprozess eines Textes mit eigenen Motiven hinzuweisen und die teils gravierende Einflussnahme der Grimms auf die Märchen zu beobachten. So kann mit Blick auf die erste Auflage der Kinder- und Hausmärchen von 1812 untersucht werden, wie dort noch der König selbst die Verse des Rumpelstilzchens belauscht und an die Müllerstochter weitererzählt (Abb. 7); eine Gestaltungsentscheidung, die Wilhelm Grimm in der zweiten Auflage von 1819 revidiert. Auch die »Vorrede« kann ein lohnenswerter Untersuchungsgegentand sein, da sich hier das bemerkenswerte editorische Selbstverständnis der Grimms als Herausgeber manifestiert. Die historische Spurensuche nach immanent präsenten »VorsprecherInnen « (wie Wilhelm Grimms spätere Ehefrau Dortchen Wild oder Mademoiselle Marie-Jeanne L'Héritier de Villandon) kann dann sogar noch weiter fortgesetzt werden.

Nach der literaturhistorischen Betrachtung kann sich der Blick in die unmittelbare Gegenwart richten, etwa indem eine aktuelle Karikatur analysiert wird, die den früheren amerikanischen Präsidenten Trump als Rumpelstilzchen persifliert (Abb. 8). Hierdurch sollen die SuS nachvollziehen, wie ein Text durch neue Reaktualisierun-

Abb. 7 Faksimile der ersten Auflage (König statt Bote) (Quelle: Brüder Grimm: Kinder- und Haus-Märchen. Große Ausgabe. Band 1, 1. Auflage, Berlin 1812, S. 253-255)

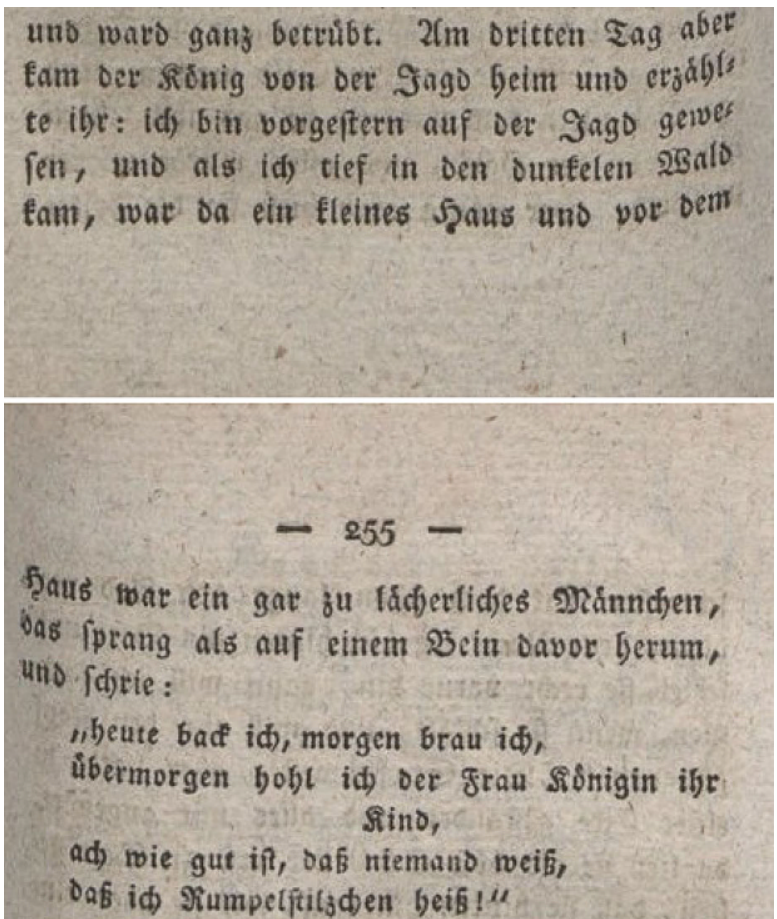


Abb. 8 Karikatur (Quelle: Ralf Böhme (RABE): Trumpelstilzchen vom 20. Juli 2016. Online auf https://de.toonpool. com/cartoons/Rumpelstilzchen 274010)

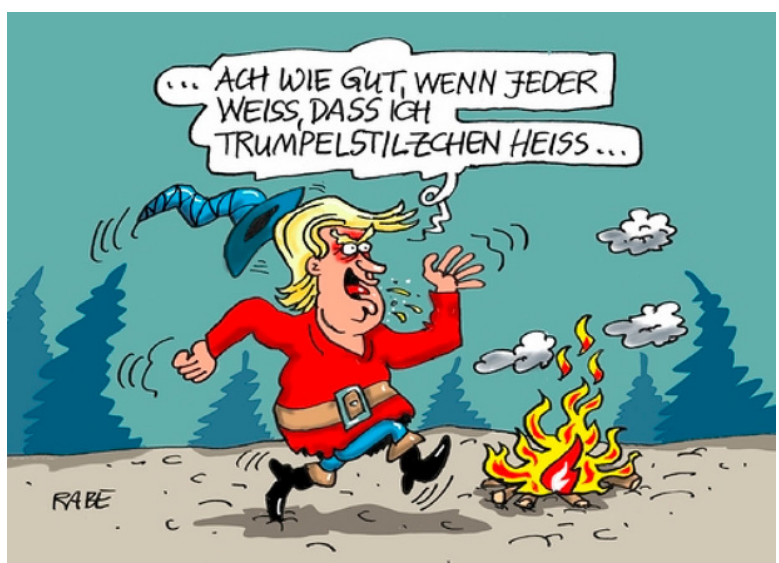

gen und Adaptionen auch neue Bedeutungsdimensionen erhält und alte latent weiter transportiert.

Bei der Suche nach heimlichen MitsprecherInnen sollte sich die Lehrkraft nicht zuletzt auch selbst ins Visier nehmen, da sie die Verse im Unterricht einsetzt und sie damit wiederum als Anschauungsmaterial zweckentfremdet und umdeutet. Die Offenlegung dieses Zusammenhangs kann wiederum Anlass bieten, mit den SuS auch deren eigene Rolle und Situation im Bildungssystem zu reflektieren. Womöglich revidieren sie dabei den Eindruck, Literaturunterricht hätte nichts mit ihrem Leben zu tun.

\section{Fazit}

Das Beispiel verdeutlicht, wie ein sprachbewusster und mediensensibler Literaturunterricht konzipiert sein könnte, um integrativ eine Medienreflexionskompetenz im angedachten übergreifenden Sinne auszubilden. Der Literaturunterricht profitiert dabei vom evidenten Lebensnäheversprechen der Sprach- und Medienkompetenz, wie auch von innovativen sprach- und medientheoretischen Beschreibungsmethoden. Gleichzeitig kann er seine methodische Sorgfalt in der textnahen Analyse voll ausspielen und seine genuine Erfahrung im Umgang mit weichen Einsichten abseits von richtig und falsch als sachgerechte Verfahrensweisen vorstellen. Literatur erscheint so umso deutlicher als überzeugendes Angebot für (Selbst-)Reflexion und Erkenntnis.

Funding Open Access funding enabled and organized by Projekt DEAL.

Open Access Dieser Artikel wird unter der Creative Commons Namensnennung 4.0 International Lizenz veröffentlicht, welche die Nutzung, Vervielfältigung, Bearbeitung, Verbreitung und Wiedergabe in jeglichem Medium und Format erlaubt, sofern Sie den/die ursprünglichen Autor(en) und die Quelle ordnungsgemäß nennen, einen Link zur Creative Commons Lizenz beifügen und angeben, ob Änderungen vorgenommen wurden. 
Die in diesem Artikel enthaltenen Bilder und sonstiges Drittmaterial unterliegen ebenfalls der genannten Creative Commons Lizenz, sofern sich aus der Abbildungslegende nichts anderes ergibt. Sofern das betreffende Material nicht unter der genannten Creative Commons Lizenz steht und die betreffende Handlung nicht nach gesetzlichen Vorschriften erlaubt ist, ist für die oben aufgeführten Weiterverwendungen des Materials die Einwilligung des jeweiligen Rechteinhabers einzuholen.

Weitere Details zur Lizenz entnehmen Sie bitte der Lizenzinformation auf http://creativecommons.org/ licenses/by/4.0/deed.de.

\section{Literatur}

Abraham, Ulf/Kepser, Matthis: Literaturdidaktik Deutsch. Eine Einführung. 4. völlig neu bearbeitete und erweiterte Auflage. Berlin 2016 [2005].

de Saussure, Ferdinand: Grundfragen der allgemeinen Sprachwissenschaft. Berlin 2001 [1916].

Dockhorn, Klaus: Macht und Wirkung der Rhetorik; vier Aufsätze zur Ideengeschichte der Vormoderne. Bad Homburg v.d. H. 1968.

Eco, Umberto: Einführung in die Semiotik. München 1972 [1968].

Ehlich, Konrad: »Medium Sprache.« In: Hans Strohner/Lorenz Sichelschmidt/Martina Hielscher (Hg.): Medium Sprache. Frankfurt am Main/New York 1998, S. 9-23.

Fauconnier, Gilles/Turner, Mark: The way we think. Conceptual Blending and the Mind's hidden Complexities. New York 2002.

Grice, Paul: »Logic and conversation. « In: Peter Cole/Jerry L. Morgan (Hg.): Syntax and Semantics 3: Speech acts. New York 1975, S. 41-58.

Iser, Wolfgang: Der Akt des Lesens. Theorie ästhetischer Wirkung. München 1984 [1976].

Jäger, Ludwig: »Sprache als Medium. Über die Sprache als audio-visuelles Dispositiv des Medialen. « In: Horst Wenzel (Hg.): Audiovisualität vor und nach Gutenberg. Wien 2001, S. 19-43.

Jäger, Ludwig: »Medialität.« In: Ekkehard Felder/Andreas Gardt (Hg.): Handbuch Sprache und Wissen. Berlin 2015, S. 106-122.

Krämer, Sybille: »Was haben >Performativität< und >Medialität< miteinander zu tun? Plädoyer für eine in der >Aisthetisierung < gründende Konzeption des Performativen.« In: Sybille Krämer (Hg.): Performativität und Medialität. München 2004, S. 13-33.

Kultusministerkonferenz: Strategie der Kultusministerkonferenz »Bildung in der digitalen Welt«. Berlin 2017 [2016].

Lösener, Hans: »Die Präzisierung der Subjektivität beim literarischen Lernen.«In: Leseräume. Zeitschrift für Literalität in Schule und Forschung 2 (2015), H.2, S. 72-84.

Maturana, Humberto R.: Erkennen: Die Organisation und Verkörperung von Wirklichkeit: ausgewählte Arbeiten zur biologischen Epistemologie. Braunschweig 1982.

Ministerium für Schule und Weiterbildung des Landes Nordrhein-Westfalen: Kernlehrplan für die Sekundarstufe II Gymnasium/Gesamtschule in Nordrhein-Westfalen: Deutsch. Düsseldorf 2014.

Peirce, Charles S.: Phänomen und Logik der Zeichen. Frankfurt am Main 1983 [1903].

Rosebrock, Cornelia/Nix, Daniel: Grundlagen der Lesedidaktik und der systematischen schulischen Leseförderung. Hohengehren 2008.

Schneider, Jan Georg: Spielräume der Medialität. Linguistische Gegenstandskonstitution aus medientheoretischer und pragmatischer Perspektive. Berlin/New York 2008.

Searle, John R.: Ausdruck und Bedeutung. Untersuchungen zur Sprechakttheorie. Frankfurt am Main 1990 [1979].

Seel, Martin: »Bestimmen und Bestimmen lassen. Anfänge einer medialen Erkenntnistheorie.« In: DZPhil 46 (1998), S. 351-365.

Shannon, Claude E./Weaver, Warren: »A Mathematical Theory of Communication. « In: Bell System Technical Journal 27 (1948), H.3, S. 379-423.

Stockwell, Peter: Cognitive Poetics. An Introduction. London/New York 2002.

Willand, Marcus: Lesermodelle und Lesertheorien. Historische und systematische Perspektiven. Berlin/ Boston 2014.

Zimmermann, Thomas Ede: Einführung in die Semantik. Darmstadt 2014.

Zymner, Rüdiger: Lyrik. Umriss und Begriff. Münster 2009. 